\title{
Writing vortex memory bits using electric field
}

\author{
G. A. Meshkov", A. P. Pyatakov",**, A. D. Belanovsky ${ }^{* *}$, K. A. Zvezdin ${ }^{* *}$ and A. S. Logginov ${ }^{*}$ \\ *Department of Physics, M.V. Lomonosov Moscow State University, Leninskie gori, Moscow 119992, Russia \\ *A. M. Prokhorov General Physics Institute, 38, Vavilova St., Moscow 119991, Russia
}

\begin{abstract}
Magnetic inhomogeneities in dielectrics can be associated with certain electric polarization distribution since they locally reduce the symmetry of the magnetic crystal. This phenomenon known as spin flexoelectricity provides a way to electrically control micromagnetic structure. Our numerical simulations show that magnetic vortexes can be nucleated and stabilized in a magnetic nanodot by static electric field. The process of nucleation and dissipation of vortices is hysteretic. A material can be found, where both vortex and homogeneous states are stable in zero electric field, which provides a basis for an electrically controlled magnetic memory device.
\end{abstract}

Key words: spin flexoelectricity, magnetic vortex, vortex memory, spiral multiferroics, inhomogeneous magnetoelectric effect.

\section{Introduction}

Magnetic vortexes and their dynamics have been intensively studied during the recent years. This interest is largely caused by further reduction of magnetic memory cell size and introduction of patterned media. Magnetic particles of small sizes display spontaneous vortex-like magnetization configurations. The control of vortex characteristics such as the chirality ${ }^{1)}$ and the polarity of the core ${ }^{2-5)}$ have been proposed. However all this techniques imply high current density and energy losses.

There is a need for a current-free vortex switching method. The spin flexoelectric effect (also known as inhomogeneous magnetoelectric effect)6) is quite promising for this application. Due to this effect the static electric field acts on magnetic inhomogeneities, as was experimentally proved on magnetic domain walls in iron garnet films ${ }^{7}$. Phenomenologically this effect can be explained as follows: the inhomogeneity of the magnetization distribution locally reduces the symmetry of the crystal. Certain types of micromagnetic structures (e.g. Neel-type magnetic domain walls) violate the inversion symmetry and allow the presence of electric polarization ${ }^{8,9}$. Microscopic mechanism of the ferroelectric polarization associated with spatially modulated spin structure can be interpreted in terms of inverse Dzyaloshinskii-Moriya interaction: the noncollinearity of spins of the adjacent magnetic ions leads to the polar displacement of the ligand ion (e.g. oxygen in the case of magnetic oxides) ${ }^{10}$. This is a common scenario of ferroelectricity in the so-called spiral multiferroics ${ }^{11)}$, i.e. the magnetic media whose electric polarization is induced by the spatially modulated spin structures.

Vortex, being an inhomogeneity too, should also be influenced by this effect ${ }^{11,12}$. The aim of this paper is to prove that a magnetic vortex state of a particle can be stable in electric field, even if spontaneous distribution is homogeneous. The particle size threshold dividing the homogeneous state and vortex or multidomain states is governed by competition of exchange and magnetostatic interactions. For low sizes of the particle the exchange prevails, providing a homogeneous state, while for bigger sizes vortex and multidomain states become more favorable. For metals like Co, this boundary lies at about $100 \mathrm{~nm}$ in diameter, but for ferrimagnetic dielectrics the saturation magnetization $\mathrm{Ms}_{\mathrm{s}}$ is usually much lower, so that the homogeneous state remains stable up to several microns.

However, in magnetic dielectrics the spin flexoelectricity can compete with exchange, and vortex state will be stable even in submicron particles. Our micromagnetic simulations show, that because of this effect vortex state can be switched by an external electric field.

\section{Methods}

We have conducted our micromagnetic simulation using a home-made micromagnetic package, SpinPM ${ }^{13)}$, which was modified to include the spin flexoelectric contribution to the free energy:

$$
f=\gamma E_{i}\left(m_{i} \partial_{j} m_{j}-m_{j} \partial_{j} m_{i}\right)
$$

where $\mathbf{E}$ is electric field, $\mathbf{m}$ is unit vector of magnetization, $\gamma$ is a material-specific constant, $\partial$ is a partial derivative sign. Indexes denote component of the vector along Cartesian axes, repeated index implies a summation. In ferroelectrics theory this form is known as Lifshitz-type invariant.

In order to use this term in micromagnetic simulation, one needs to calculate its contribution to the effective magnetic field ${ }^{14)}$ :

$$
\begin{aligned}
& H_{M E i}=-\frac{\delta f}{\delta M_{i}}= \\
& =\frac{\gamma}{M_{s}}\left[2\left(E_{i} \frac{\partial m_{j}}{\partial x_{j}}-E_{j} \frac{\partial m_{j}}{\partial x_{i}}\right)-m_{j}\left(\frac{\partial E_{j}}{\partial x_{i}}-\frac{\partial E_{i}}{\partial x_{j}}\right)\right]
\end{aligned}
$$


where $M_{S}$ is spontaneous magnetization.

One can see, that in the presence of electric field a magnetic field will arise in inhomogeneities (where $\partial \mathbf{m} / \partial \mathbf{x} \neq 0$ ). However, when the magnetization is homogeneous there will be no effect: the first term in parentheses (eq.2) will be zero because magnetization is homogeneous and the second term, which is $\mathbf{m} \cdot \operatorname{curl}(\mathbf{E})$, will also be zero in the conservative electric field.

We have conducted a micromagnetic simulation of a full life cycle of a vortex in a nanoparticle: nucleation, stabilization and transformation/dissipation, using static electric field as a controlling force. The size of the mesh was chosen as small as $2 \mathrm{~nm}$.

The saturation magnetization of the material was varied in the range $M_{\mathrm{s}}=5-50 \mathrm{G}$, the exchange stiffness constant was $\mathrm{A}=3^{*} 10^{-7} \mathrm{erg} / \mathrm{cm}$, and the easy-plane anisotropy was set to $-10^{5} \mathrm{erg} / \mathrm{cm}^{3}$. These parameters are typical for magnetic dielectrics with high magnetic ordering temperature, like bismuth ferrite $\mathrm{BiFeO}_{3}$ or iron garnets $\mathrm{R}_{3} \mathrm{Fe}_{5} \mathrm{O}_{12}$ ( $\mathrm{R}=$ rare earth). These are the materials with the most pronounced spin flexoelectric properties: in $\mathrm{BiFeO}_{3}$ the ferroelectric polarization induces spin modulation ${ }^{15}$, in $\mathrm{R}_{3} \mathrm{Fe}_{5} \mathrm{O}_{12}$ the Neel-type magnetic domain walls are electrically polarized ${ }^{7}$. The constant $\gamma$ that characterizes this inhomogeneous magnetoelectric interaction can be estimated as $\left.10^{-6} \sqrt{\mathrm{erg} / \mathrm{cm}} 7,15\right)$.
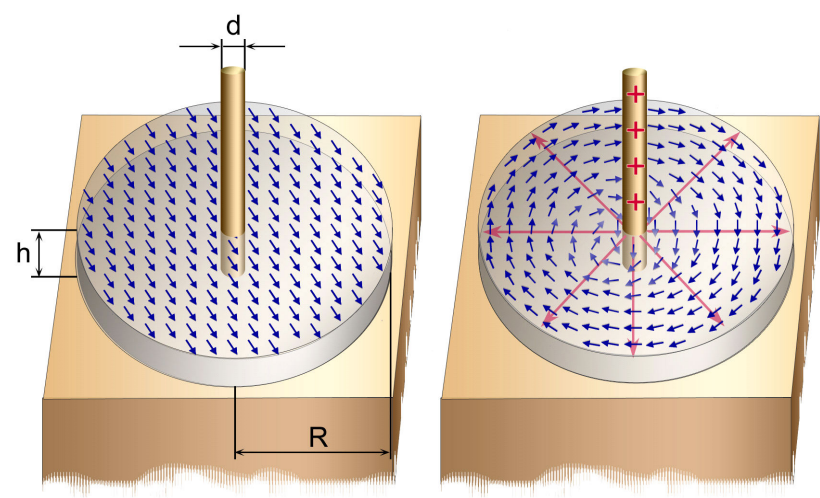

Fig. 1 The geometry of the problem. The magnetic nanodot is electrically polarized with the wire electrode. $\mathrm{d}=5 \mathrm{~nm}, \mathrm{R}=120 \mathrm{~nm}, \mathrm{~h}=10 \mathrm{~nm}$.

a) homogeneous magnetization distribution b) vortex structure

The size of the magnetic particle was chosen $\mathrm{R}=120$ $\mathrm{nm}$ that is an order of the exchange length $\sqrt{\mathrm{A} / M_{\mathrm{s}}^{2}}$ for the magnetization $M_{\mathrm{s}}<50 \mathrm{G}$, so that the spontaneous magnetic state of the particle was the homogeneous one in this case.

The control electric field was chosen as a field produced by a 5-nm diameter wire, going along the $\mathrm{Z}$ axis through the center of the particle. It falls off as $1 /|r|$ with distance from the wire. In the absence of electric field the magnetization in the particle is almost homogeneous, because demagnetizing field energy is much less than exchange energy. As follows from (eq.2) in a particle with homogenous magnetization distribution effective magnetic field would be zero, so we have introduced an anisotropy defect in a portion of a particle to get a perturbation. The defect was the area with an easy-axis anisotropy of $10^{5} \mathrm{erg} / \mathrm{cm}^{3}$ while it remains easy-plane one in the rest of the particle.

\section{Results}

Figure 2 shows magnetization distribution of the nanoparticle in the vortex state. The effective field distribution of magnetoelectric origin (2) stabilizes vortex state while the effective field of exchange interaction tries to unwind the vortex (effective fields are shown in the insets of Fig. 2).

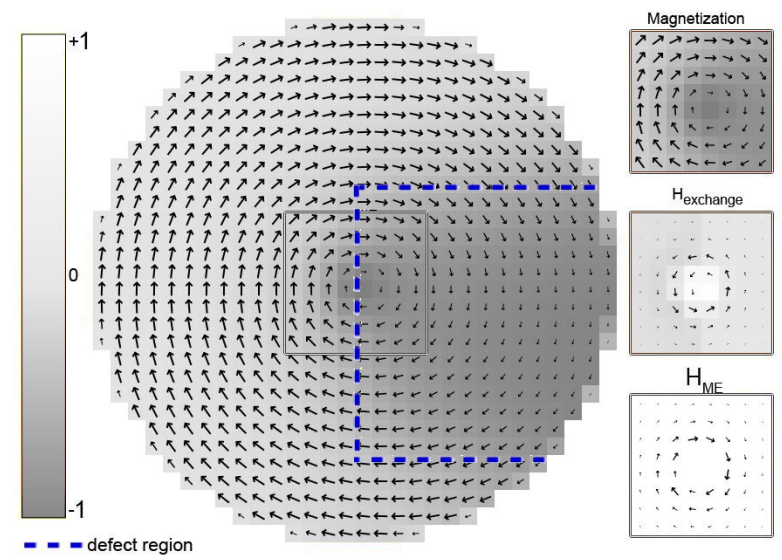

Fig. 2 The magnetization distribution in the magnetic nanodot. The insets show the magnetization and effective fields distributions in the central area of the vortex (vortex core): Hexchange is effective exchange field, $\mathrm{H}_{\mathrm{ME}}$ is effective magnetoelectric field in equation (2). The area selected with dashed line is the region of the particle with easy axis anisotropy (the defect). The $\mathrm{x}, \mathrm{y}^{-}$components of the vectors are presented with arrows while z-component is represented by color. Each cell on the figure corresponds to an average of a $2 \times 2$ block of calculation cells.

Figure 3 shows the dependence of winding number (WN) of particle's magnetization on the electric field strength. Winding number is an integer showing how many times the magnetization rotates by $180 \mathrm{deg}$ if one follows a path circumscribing the center of the particle. As the field increases to some "nucleation" value, the vortex $(\mathrm{WN}=1)$ becomes the favorable state and nucleates from a disturbed homogeneous state $(\mathrm{WN}=0)$. It remains stable in higher fields. As the field falls below some critical value (smaller than nucleation field), the vortex state returns to homogeneous one (vortex core is pushed out of the particle). 
In materials with higher saturation magnetization (and therefore higher stray fields) the vortex state becomes more favorable, so one needs lower electric field to nucleate a vortex (fig.3 dotted line). In the material with $M_{\mathrm{s}}=150 \mathrm{G}$, the energy of the vortex state becomes so low that the vortex unwinding field is negative and in zero electric field one can get two metastable states: homogeneous state and vortex one. This creates an opportunity of switching vortex memory cells with static electric field, which is much more energy efficient than current-driven switching. The stray field of a homogeneous state is much higher than that of a vortex state, so the reading of such a memory cell can be done using a GMR device.

It should be noted that this effect is universal and can appear in any magnetic dielectric material, even in the centrosymmetric one ${ }^{9)}$. The electric field induced magnetic switching is difficult to implement for magnetic metals because in the bulk of the conducting material the electric field is screened by the charge carriers. However recent experiments ${ }^{16)}$ show that in the case of single layer metal the magnetoelectric effect can still be observed.

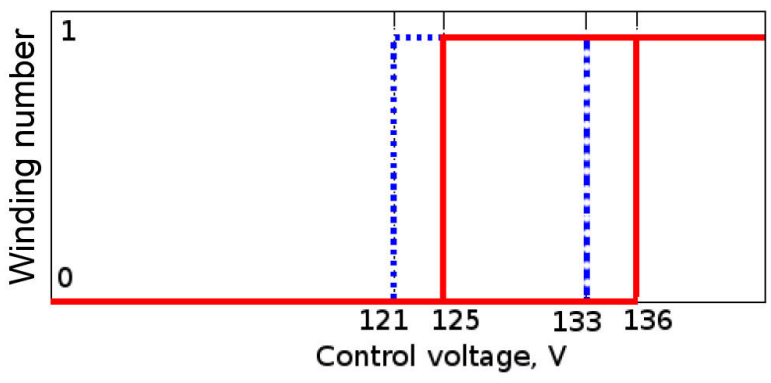

Fig. 3 The hysteretic dependence of winding number on electric voltage. The solid line corresponds to the magnetization $M_{\mathrm{s}}=5 \mathrm{G}$, the dotted line corresponds to $M_{\mathrm{s}}=50 \mathrm{G}$.

\section{Conclusion}

We have shown that the magnetization in a submicron dot can be switched by electric field between two metastable states: the homogeneous state and the vortex one. To create an effective magnetic field no current is needed and therefore this type of magnetization switching is energy efficient. Due to the hysteretic character of switching metastable states can exist in the same electric field depending on the history of switching. By selecting an appropriate material we can get a particle in which both state are possible in zero electric field. Such a particle can form a basis of an electrically controlled memory device, with homogenous and vortex states representing " 0 " and " 1 ".

Acknowledgements Authors are grateful to Anatoly K. Zvezdin for the interest to the work and valuable discussions. The work is supported by RFBR grant 10-02-13302-RT-omi and 10-02-90475-Ukr_a .

\section{References}

1) M. Tanase, A. K. Petford-Long, O. Heinonen, K. S. Buchanan, J. Sort, and J. Nogués: Phys. Rev. B, 79, 014436 (2009).

2) K. Yamada, S. Kasai, Y. Nakatani, K. Kobayashi, H. Kohno, A. Thiaville, and T. Ono: Nat Mater, 6, 270-273 (2007).

3) T. Okuno, K. Shigeto, T. Ono, K. Mibu, and T. Shinjo: $J$. Magn. Magn. Mater., 240, 1 (2002).

4) A. Thiaville, J. M. García, R. Dittrich, J. Miltat, and Th. Schrefl: Phys. Rev.B, 67, 094410 (2003).

5) A. V. Khvalkovskiy, A. N. Slavin, J. Grollier, K. A. Zvezdin, and K. Yu. Guslienko: Appl. Phys. Lett., 96, 022504 (2010).

6) A. P. Pyatakov, and A. K. Zvezdin: The European Physical Journal B - Condensed Matter and Complex Systems, 71, 419-427 (2009).

7) A. S. Logginov, G. A. Meshkov, A. V. Nikolaev, E. P. Nikolaeva, A. P. Pyatakov, and A. K. Zvezdin: Appl. Phys. Lett, 93, 182510 (2008).

8) V. G. Bar'yakhtar, V. A. L'vov, and D. A. Yablonskii: JETP Lett., 37, 673 (1983).

9) I. Dzyaloshinskii: $E P L, \mathbf{8 3}, 67001$ (2008).

10) I. A. Sergienko, and E. Dagotto: Phys. Rev. B, 73, 094434 (2006).

11) M. Mostovoy: Phys. Rev. Lett., 96, 067601 (2006).

12) A. Pyatakov, G. Meshkov, and A. Logginov: Moscow University Physics Bulletin, 65, 329-331 (2010).

13) A. V. Khvalkovskiy, J. Grollier, N. Locatelli, Y. V. Gorbunov, K. A. Zvezdin and V. Cros: Appl. Phys. Lett., 96, 212507 (2010).

14) T. Fischbacher, M. Franchin, and H. Fangohr: J. Appl. Phys., 109, 07D352 (2011).

15) Yu. F. Popov, A. K. Zvezdin, G.P Vorob'ev, A. M. Kadomtseva, V. A. Murashev, and D. N. Rakov: JETP Lett., 57, 69 (1993).

16) L. Gerhard, T. K. Yamada, T. Balashov, A. F. Taka'cs, R. J. H. Wesselink, M. Da“ne, M. Fechner, S. Ostanin, A. Ernst, I. Mertig and W. Wulfhekel: Nature Nanotechnology, 5, 792 (2010).

Received June 7, 2011; Revised July 29, 2011;

Accepted August 27, 2011. 